\title{
Desigualdades en salud y en la utilización y el desempeño de los servicios sanitarios en las Comunidades Autónomas
}

\author{
Anna García-Altés, Maica Rodríguez-Sanz, \\ Glòria Pérez, Carme Borrell \\ Agència de Salut Pública de Barcelona
}

\section{Resumen}

El estudio de las desigualdades sociales en salud aumentó en España en la década de los 90, existiendo actualmente un importante conjunto de investigaciones que analizan las desigualdades en el estado de salud, la utilización de servicios sanitarios y la mortalidad, así como un número elevado de estudios basados en áreas geográficas pequeñas.

Este artículo resume las principales conclusiones de tres informes realizados por encargo del Observatorio del SNS sobre las desigualdades en salud de género, clase social y geográficas en España. En particular, los objetivos del artículo son describir las desigualdades según clase social en el estado de salud percibido, la utilización y el desempeño de los servicios sanitarios, y la mortalidad global en los hombres y las mujeres de las CCAA.

Se trata de un estudio ecológico de corte transversal. La unidad de análisis fueron las 18 CCAA de España. A través del Ministerio de Sanidad y Consumo, fueron consultadas la Encuesta Nacional de Salud (2003), el Registro de mortalidad (2000-2002) y el Registro de altas hospitalarias (CMBD-AH, 2002). Se seleccionaron los siguientes indicadores: \% de población que declara mal estado de salud, $\%$ de población que declara haber visitado un médico/a general y especialista, tasa de hospitalización por cirugía de fractura de fémur, por accidente vascular cerebral, por enfermedad pulmonar obstructiva crónica, tasa de hospitalización urgente por cáncer de pulmón, y tasa de mortalidad estandarizada. Los indicadores se describieron para cada CCAA, separando hombres y mujeres, expresados como porcentajes y tasas estandarizadas por edad. En el caso de los indicadores de estado de salud percibido y utilización de servicios sanitarios, éstos se analizaron según clase social.

Los resultados de nuestro estudio muestran que existen desigualdades de género y clase social en el estado de salud, la utilización de servicios sanitarios, el desempeño de los servicios sanitarios y la mortalidad entre las CCAA de España. En todas las CCAA, el estado de salud percibido de las mujeres es peor que el de los hombres, y existen desigualdades geográficas. En cuanto al uso de servicios sanitarios, también se observa una distribución geográfica desigual en las CCAA, tanto debida a la distribución desigual de la salud en las CCAA, como a aspectos relacionados con el sistema sanitario. En el desempeño de los servicios sanitarios, las diferencias en las tasas de hospitalización entre CCAA y sexos pueden ser un indicativo de desigualdades en el acceso a los servicios hospitalarios. Finalmente, la mortalidad global es siempre superior en los hombres que en las mujeres, excepto para algunas causas concretas, existiendo también diferencias entre CCAA. Los resultados también permiten ver algunas CCAA que merecen una consideración especial.

Los resultados contribuyen a generar evidencia sobre la magnitud del problema en nuestro país y los posibles beneficios que pueden derivarse de su estudio. Esta información puede ser la base para el desarrollo de políticas posteriores dirigidas a la reducción de desigualdades, tanto desde el punto de vista de la salud pública y la política sanitaria, como de otras políticas sociales.

Palabras clave: Desigualdades, estado de salud, mortalidad, desempeño de los servicios sanitarios. Clasificación JEL: I10 


\begin{abstract}
The study of social inequalities in health in Spain increased during the 90's. Nowadays there is an important body of research about inequalities in health status, utilization of health care services and mortality, as well as a number of studies based on small areas.

This paper is an abstract of the main conclusions of three reports done for the Spanish NHS Observatory about gender, social class, and geographical inequalities in health. In particular, the objectives of the paper are to describe social class inequalities in perceived health status, utilization and performance of health care services, and overall mortality in men and women of Spain regions.

This is a cross-sectional ecological study. Study units were the 18 regions of Spain. The Spanish Ministry of Health provided us the National Health Survey (2003), the Mortality Register (2000-2002), and the Hospital Discharge Register (2002). The following indicators were selected: \% population with perceived bad health status, \% population that has visited a general practitioner and specialist, hospitalization rate for femur fracture, cerebrovascular accident, chronic obstructive pulmonary disease, and emergent hospitalization for lung cancer, and standardized mortality rate. For all regions, indicators were described separately for men and women, and age standardized percentages and rates were calculated. Indicators of perceived health status and utilization of healthcare services were analyzed by social class.

The results of our study show gender and social class inequalities in perceived health status, utilization and performance of health care services, and mortality among Spanish regions. In all regions, perceived health status among women is worse than among men, and there are geographical inequalities. Regarding utilization of health care services, there is an unequal distribution among regions related with the unequal distribution of health, and factors related with the health care system. In the performance of health care services, the differences in hospitalization rates among regions and genders could be an indicator of access inequalities to health care services. Finally, overall mortality is always higher among men than among women, except for a few causes, with differences among regions. The results also allow pointing out some regions that deserve special attention.

Those results contribute to generate evidence about the issue and about the potential benefits of further research. This information could be the base for the development of policies to address inequalities, both from the point of view of public health and health policy, as well as from other social policies.
\end{abstract}

Key words: Inequalities, health status, mortality, perfomance of healthcare services.

JEL Classification: I10

\title{
Antecedentes
}

El estudio de las desigualdades sociales en salud aumentó en España en la década de los 90, existiendo actualmente un importante conjunto de investigaciones que analizan las desigualdades en el estado de salud, la utilización de servicios sanitarios y la mortalidad. Entre ellos cabe mencionar un número elevado de estudios basados en áreas geográficas pequeñas cómo los distritos, los barrios o las zonas básicas de salud de algunas ciudades entre las que destaca muy especialmente Barcelona (1).

En el año 1994, Regidor et al. publicaron un libro exhaustivo sobre las desigualdades en salud en España, utilizando tanto datos de mortalidad como datos de diferentes encuestas de salud (2). Los investigadores analizaron las diferencias y desigualdades en el estado de salud, la mortalidad, la esperanza de vida, la esperanza de vida libre de incapacidad, el estado de salud percibido, la altura, el peso al nacer y algunos problemas de salud prioritarios (enfermedad isquémica del corazón, enfermedad cerebrovascular, cáncer de pulmón, accidentes de tráfico, sida, consumo de tabaco y de alcohol y obesidad). 
Posteriormente, en 1996 el Ministerio de Sanidad y The School of Hygiene and Public Health de la Johns Hopkins University (EEUU) editaron un libro realizado por un grupo de expertos sobre las desigualdades sociales en salud en España. En él, se analizaban las desigualdades según clase social ocupacional, edad, sexo y comunidad autónoma (CCAA) con datos de las encuestas de salud, y se describió la evolución de las desigualdades en lo referente al estado de salud, las conductas relacionadas con la salud y la utilización de servicios sanitarios (3).

Si bien hay evidencia sobre las desigualdades en el acceso y la utilización de servicios sanitarios en España, analizadas mayoritariamente a partir de encuestas de salud, no se conocen las desigualdades existentes en el desempeño de los servicios sanitarios $(4,5,6,7,8)$. De hecho, los servicios sanitarios son sólo uno de los determinantes de la salud, si bien es el determinante al que el presupuesto sanitario dedica más recursos económicos en los países desarrollados. La medida del desempeño de los servicios sanitarios está recibiendo una atención creciente debido, por una parte, a las presiones fiscales ante un gasto sanitario creciente y, por otra, al aumento de las expectativas y exigencias de la población $(9,10,11,12)$. Así pues, conocer hasta que punto existen desigualdades en la consecución de los objetivos que se proponen los servicios sanitarios es un hecho legítimo, además de ser una manera de rendir cuentas ante la sociedad y una fuente de información con la que diseñar políticas sanitarias (13).

Los registros de mortalidad son otra fuente de información de mucha utilidad para analizar desigualdades en salud entre CCAA, ya sea en mortalidad global o por causas $(14,15)$. Los primeros estudios realizados en España que establecieron las desigualdades en mortalidad se realizaron en Barcelona, al mismo tiempo que se analizaban las desigualdades en ocho provincias con buena calidad en la información de la ocupación en las estadísticas vitales $(16,17,18,19)$. Estos estudios, que se realizaron a partir de datos individuales, no se han podido reproducir para el resto de España por la carencia de la mencionada información. Sin embargo, en diversos estudios ecológicos se ha podido establecer la desigual distribución de la mortalidad por CCAA según indicadores de privación material para distintos periodos temporales. Entre éstos cabe destacar los informes realizados en 1996, 1998 y más recientemente, el atlas de mortalidad por áreas pequeñas $(20,21)$. En todos ellos se obtuvo un gradiente noreste-suroeste de las variables utilizadas para estimar la privación material (el desempleo, el analfabetismo, la clase social y el hacinamiento) que se repite en la mortalidad (razón de mortalidad comparativa y esperanza de vida al nacer) y que se observa de nuevo cuando se asocia la mortalidad y la privación material, de forma que en las CCAA del sur del país, las más desfavorecidas, se produce un importante exceso del número de fallecimientos respecto al resto de CCAA. En cambio, otros indicadores como los de mortalidad prematura presentaron desigualdades de género y por grupos de edad en su patrón de distribución (22).

En la última década, diversos artículos científicos han puesto de relieve la existencia de desigualdades en salud y en las tendencias a lo largo de los años. Además, muy recientemente, se han publicado informes específicos sobre el tema. Así por 
ejemplo, el año 2005 se ha publicado el informe «L'evolució de les desigualtats en salut a Catalunya» y el Informe de la Sociedad Española de Salud Pública de 2004 analiza con profundidad las desigualdades sociales en salud $(23,24,25)$.

Este artículo resume las principales conclusiones de tres informes realizados por encargo del Observatorio del SNS sobre las desigualdades en salud de género, clase social y geográficas en España $(26,27,28)$. Estos informes se integran en la línea de estudios comparados de resultados en salud asignada al Observatorio del SNS, en el ámbito de las políticas de equidad y cohesión social. En particular, los objetivos del artículo son:

- Describir las desigualdades según clase social en el estado de salud percibido, y la utilización de servicios sanitarios, en los hombres y las mujeres de las CCAA.

- Analizar las desigualdades en el desempeño de los servicios sanitarios, en los hombres y las mujeres de las CCAA.

- Analizar las desigualdades en mortalidad global en los hombres y las mujeres de las CCAA.

\section{Metodología}

Diseño y población de estudio

Se trata de un estudio ecológico de corte transversal. La unidad de análisis fueron las 18 CCAA de España, con poblaciones entre 137.916 habitantes (Ceuta y Melilla) y 7.357 .558 habitantes (Andalucía).

\section{Fuentes de información}

A través del Ministerio de Sanidad y Consumo, fueron consultadas las siguientes fuentes de información:

- Encuesta Nacional de Salud, 2003. La ENS recoge, a través de entrevista personal directa, variables demográficas, estado de salud percibido, enfermedades crónicas, morbilidad percibida, utilización de servicios sanitarios y medicamentos, hábitos de salud, actividades preventivas y cobertura sanitaria.

- Registro de mortalidad, 2000-2002, que se alimenta de los boletines estadísticos de defunción, e incluye, entre otras, las variables de sexo, edad y causa básica de defunción, codificada según la Clasificación Internacional de Enfermedades, décima revisión.

- Registro de altas hospitalarias (CMBD-AH), 2002. El registro de altas hospitalarias es una base de datos clínico-administrativa que recoge información del 
alta de los episodios que originaron la hospitalización. Los diagnósticos y procedimientos están codificados según la codificada según la Clasificación Internacional de Enfermedades, décima revisión.

Los datos de población agregados para CCAA y según sexo y grupos de edad se han obtenido del censo de población y viviendas del año 2001 del Instituto Nacional de Estadística.

\section{Indicadores}

Para el análisis de las desigualdades en salud se han seleccionado los siguientes indicadores:

- Indicadores de estado de salud: \% de población que declara mal estado de salud.

- Indicadores de utilización de servicios sanitarios: \% de población que declara haber visitado un médico/a general y especialista en los últimos 15 días.

- Indicadores de desempeño: tasa de hospitalización por cirugía de fractura de fémur, por accidente vascular cerebral, por enfermedad pulmonar obstructiva crónica (EPOC) y tasa de hospitalización urgente por cáncer de pulmón.

- Indicadores de mortalidad: Tasa de mortalidad estandarizada.

\section{Variables}

- Sexo.

- Edad.

- Clase social, según la clasificación propuesta por la Sociedad Española de Epidemiología y agrupada en clases manuales y no manuales (29).

- Comunidad Autónoma.

\section{Análisis de datos}

Los indicadores de estado de salud percibido y visitas al médico/a general y especialista se han descrito para cada CCAA según clase social, expresados como porcentajes estandarizados por edad. Los indicadores de desempeño y mortalidad se han descrito para cada CCAA, expresados como tasas estandarizadas por edad. Los indicadores se han calculado separando hombres y mujeres y se han estandarizado por edad por el método directo, siendo la población española del censo de población y viviendas de 2001 la utilizada como población de referencia. Se han realizado mapas de cuartiles y quintiles de los indicadores para hombres y mujeres. 


\section{Resultados}

\section{Estado de salud percibido}

El año 2003 en España, un 27\% de hombres y un 36,8\% de las mujeres mayores de 15 años declararon un mal estado de salud percibido (regular, malo o muy malo), porcentajes que son bastante estables desde 1993 (26). Se observan desigualdades en la prevalencia de mal estado de salud percibido en las CCAA, siendo las personas del sur y oeste del país las que declaran peor salud (Figura 1).

\section{FIGURA 1}

\section{PREVALENCIAS DE MAL ESTADO DE SALUD PERCIBIDO, EN HOMBRES Y MUJERES MAYORES DE 15 AÑOS, EN LAS CCAA DE ESPAÑA EL AÑO 2003. PORCENTAJES ESTANDARIZADOS POR EDAD}
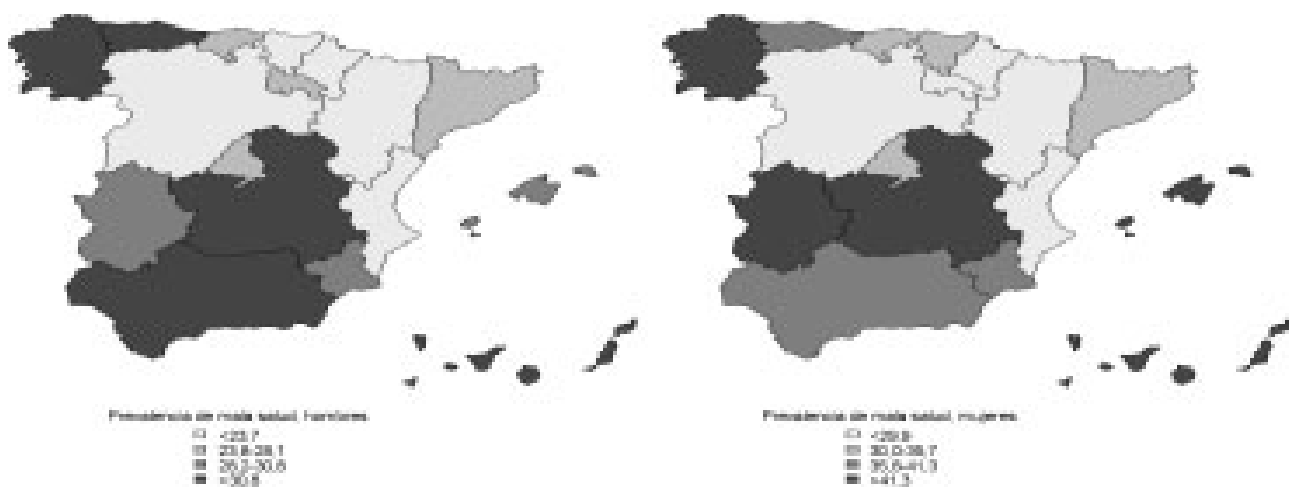

La mayoría de las CCAA presentan diferencias significativas en el estado de salud percibido según la clase social. Destacan algunas como Canarias y Galicia donde la población de clases menos favorecidas declara los mayores porcentajes de mala salud (más del 40\% de los hombres, y más del 50\% de las mujeres de clases manuales), e incluso las personas de clases más favorecidas de estas CCAA superan en las prevalencias a las personas de clases menos favorecidas del resto de CCAA. En cambio otras CCAA como Aragón o La Rioja registran prevalencias de mala salud muy inferiores y sin diferencias según la clase social (Figura 2).

\section{Visitas al médico/a general y especialista}

Haber visitado un médico/a general las dos semanas previas a la entrevista es declarado por un $17,7 \%$ de hombres y un $24,5 \%$ de mujeres mayores de 15 años en España el año 2003. Un 6,2\% de hombres y un 8\% de mujeres declararon haber acudido al médico/a especialista, aumentando las proporciones en Canarias y 
FIGURA 2

PREVALENCIAS DE MAL ESTADO DE SALUD PERCIBIDO SEGÚN LA CLASE SOCIAL EN HOMBRES Y MUJERES MAYORES DE 15 AÑOS, EN LAS CCAA DE ESPAÑA EL AÑO 2003. PORCENTAJES ESTANDARIZADOS POR EDAD
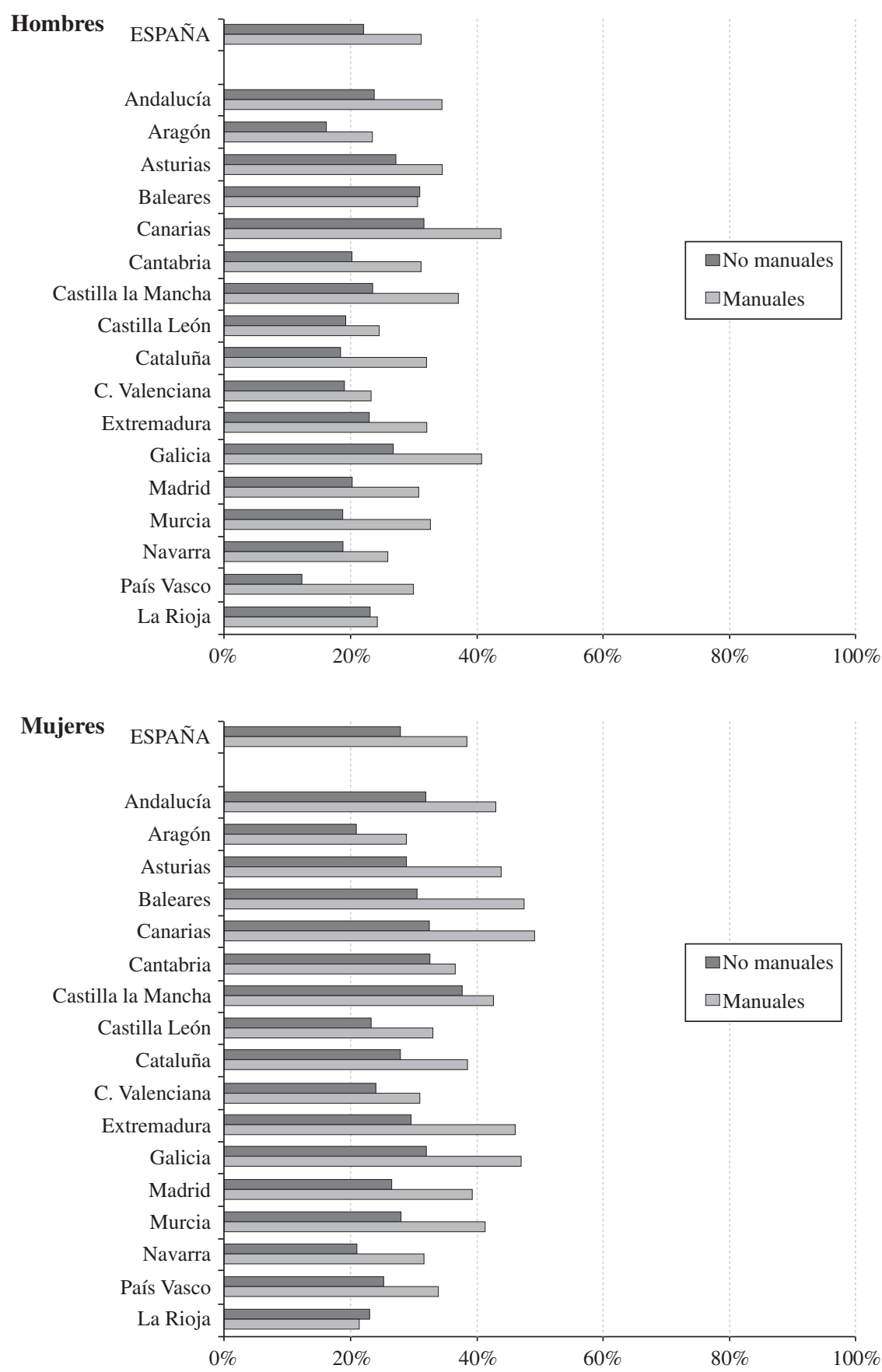
Cataluña. El año 2003 en España, existen desigualdades según género y clase social en la proporción de personas que han visitado un médico/a general. Las mujeres declaran haberse visitado más y, tanto en hombres como en mujeres, las personas de clases más favorecidas presentan proporciones inferiores de visitas al médico general (aproximadamente un 11\% en hombres y mujeres de clases no manuales) y aumentan progresivamente en las de clases menos favorecidas, sobre todo en mujeres (un 21,4\% de hombres y un 33,3\% de mujeres de clases manuales), y estas desigualdades de clase social han aumentado en ambos sexos en la última década.

La proporción de personas que visitan un especialista cambia según clase social, siendo mayor en las clases privilegiadas. Así, los hombres de clases menos favorecidas han visitado un especialista en un 4,7\%, y aumenta en los de clases más favorecidas a un $6,9 \%$. En mujeres, el patrón de desigualdad es el mismo y sigue un gradiente claro de clase social (un 6,9\% en las de clases manuales frente al 9,5\% de las de clases no manuales).

\section{Desempeño}

Las diferencias en las tasas de hospitalización entre CCAA y sexos pueden ser un indicativo de desigualdades en el acceso a los servicios hospitalarios. Así, en el caso de la cirugía de fractura de cuello de fémur, destacan Cataluña, Aragón y Andalucía (tasas alrededor de 55 por 100.000 habitantes en hombres y 155 por 100.000 habitantes en mujeres); las tasas más bajas se dan en Galicia (25,6 por 100.000 habitantes en hombres y 80,7 por 100.000 habitantes en mujeres). Para las hospitalizaciones por accidente vascular cerebral destacan las altas tasas de Murcia, Cantabria y Asturias (valores cercanos a los 200 por 100.000 habitantes) y las tasas bajas de Canarias y Castilla y León (tasas alrededor de 125 por 100.000 habitantes en ambos sexos). En el caso de la EPOC, destacan Cataluña y Asturias (550 por 100.000 habitantes en ambos sexos), mientras que los valores más bajos se dan en Canarias (133,6 y 21,3 por 100.000 habitantes, en hombres y mujeres, respectivamente) (Figura 3). Asturias y Ceuta y Melilla tienen las tasas más altas de hospitalización por cáncer de pulmón en hombres (161,5 y 155,7 por 1.000 habitantes, respectivamente); las tasas más bajas se dan en La Rioja y Canarias en hombres (66,1 y 70,3 por 100.000 habitantes, respectivamente) y Extremadura y Andalucía en mujeres ( 8,1 y 8,5 por 100.000 habitantes, respectivamente).

\section{Mortalidad global}

La tasa de mortalidad en España ha descendido considerablemente a lo largo del siglo XX. Esta reducción de la mortalidad se ha producido en todas las edades y ello se traduce en una mejora de la esperanza de vida de la población. En el año 2002 en 
FIGURA 3

TASA DE HOSPITALIZACIÓN POR EPOC, EN HOMBRES Y MUJERES, EN LAS CCAA DE ESPAÑA EN 2002. TASAS ESTANDARIZADAS POR EDAD POR 100.000 HABITANTES

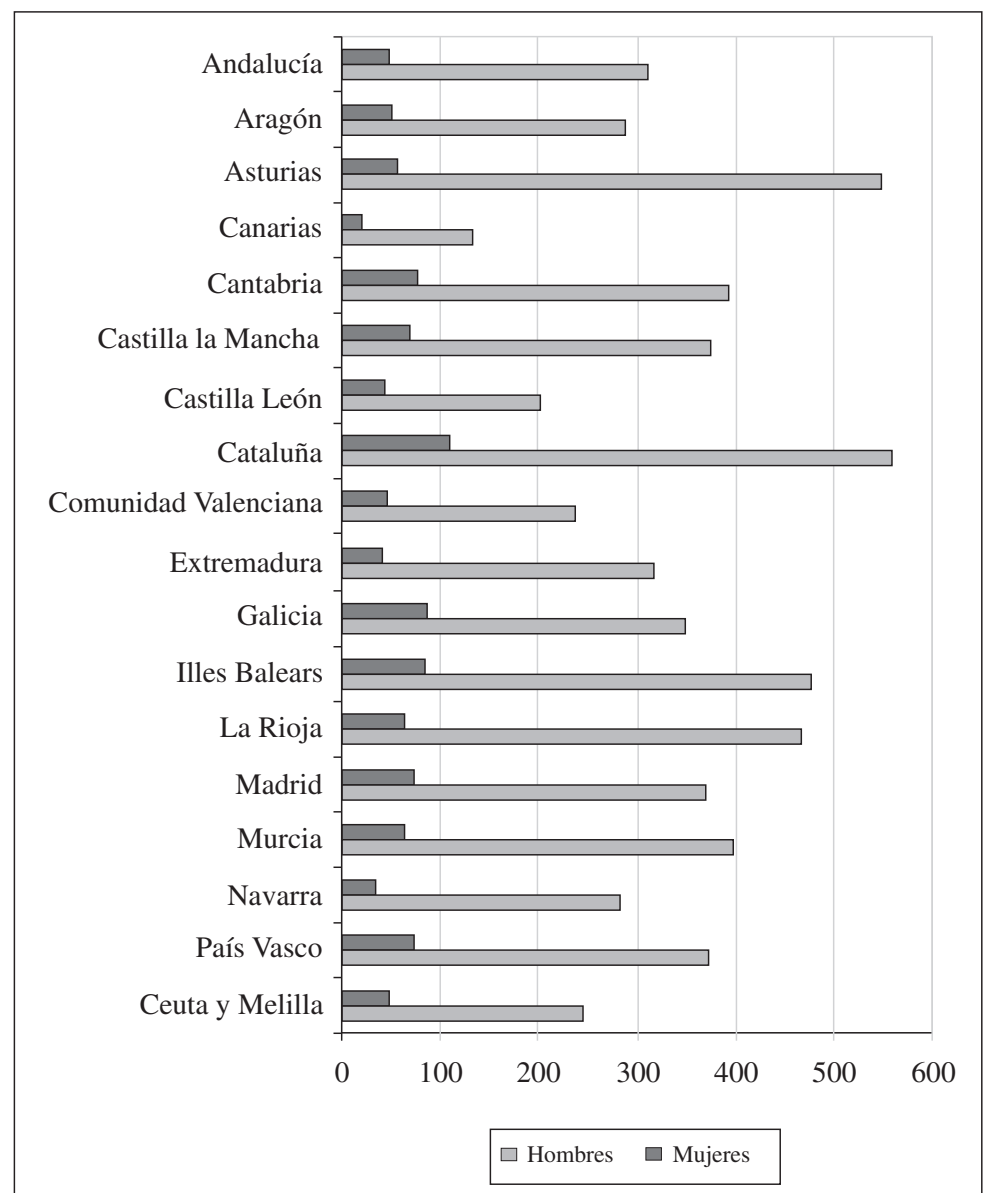

España se produjeron 368.618 defunciones (193.269 en hombres y 175.349 en mujeres). La tasa de mortalidad correspondiente es 892,2 por 100.000 habitantes $(953,7$ por 100.000 hombres y 833,1 por 100.000 mujeres). Estas cifras sitúan España entre los países con los niveles más bajos de mortalidad de entre los países de la región europea y en el mismo grupo que Inglaterra, Islandia, Suecia, Noruega, Holanda, Austria y Grecia (29).

En los años 2000-2002, las CCAA que presentan una mayor mortalidad son Ceuta y Melilla, Canarias y Andalucía en ambos sexos y las CCAA que muestran una menor mortalidad en los hombres son la Rioja, Castilla y León y Castilla-La Mancha y en las mujeres son La Rioja, Castilla y León y Navarra (Figura 4). La mortalidad global es superior en los hombres. 
FIGURA 4

DISTRIBUCIÓN POR QUINTILES DE LA MORTALIDAD GLOBAL, EN HOMBRES Y MUJERES, EN LAS CCAA DE ESPAÑA EN 2000-2002. TASAS POR 100.000 HABITANTES ESTANDARIZADAS POR EDAD
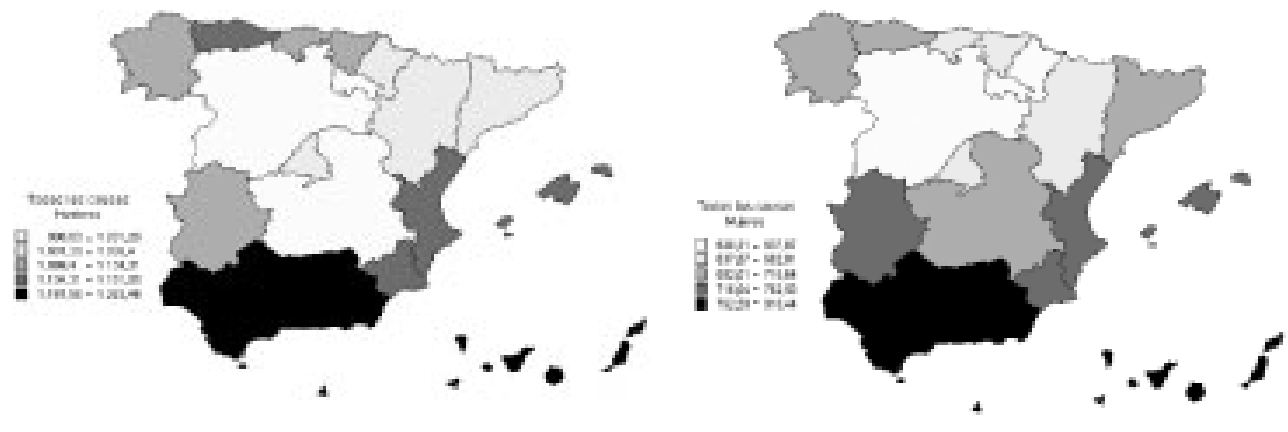

\section{Discusión}

Los resultados de nuestro estudio muestran que existen desigualdades de género y clase social en el estado de salud, la utilización de servicios sanitarios, el desempeño de los servicios sanitarios y la mortalidad entre las CCAA.

Como puede verse, en todas las CCAA, el estado de salud percibido de las mujeres es peor que el de los hombres. Adicionalmente, se observan desigualdades geográficas en el estado de salud percibido: las CCAA del sur y oeste del país, como son Canarias, Andalucía, Extremadura y Galicia presentan peores indicadores de estado de salud percibido, mientras que las CCAA del norte del país como son Navarra, La Rioja y Cantabria tienen mejores indicadores. Estas desigualdades podrían estar relacionadas con el nivel socioeconómico de las CCAA, ya que los recursos sociales, económicos y sanitarios que dispone cada región y la pobreza del área de residencia podrían tener influencia en el estado de salud de la población (30).

En cuanto al uso de servicios sanitarios, se observa una distribución geográfica desigual en las CCAA, que podría estar relacionada tanto con la distribución desigual de la salud y la enfermedad en las CCAA, como con aspectos más relacionados con el sistema sanitario como son la oferta, la accesibilidad, la calidad de los servicios y las variaciones en la práctica médica.

En relación al desempeño de los servicios sanitarios, las diferencias en las tasas de hospitalización entre CCAA y sexos pueden ser un indicativo de desigualdades en el acceso a los servicios hospitalarios. Para algunas condiciones, las tasas de hospitalización son mayores en mujeres que en hombres ( $p$. ej. cirugía de fractura de cuello de fémur), mientras que sucede lo contrario en otras (p.ej. accidente vascular cerebral, EPOC, cáncer de pulmón). Las variaciones en las tasas de hospitalización entre CCAA pueden llegar a ser de hasta tres veces (como es el caso de la EPOC en Cataluña o Asturias versus Canarias). 
Finalmente, y en referencia a la mortalidad, ésta es siempre superior en los hombres que en las mujeres, excepto para algunas causas concretas, existiendo también diferencias entre CCAA.

Los resultados también permiten ver algunas CCAA que merecen una consideración especial. Ceuta y Melilla tiene menor tasa de hospitalización por fractura de cuello de fémur, mientras que son superiores por cáncer de pulmón y hospitalizaciones evitables por diabetes y úlcera gástrica y/o duodenal. Ceuta y Melilla, Andalucía y Islas Canarias son las CCAA que con mayor frecuencia están entre las CCAA que presentan una mayor mortalidad global. En el otro extremo se sitúan Madrid, Castilla-La Mancha, Castilla y León y La Rioja que son las CCAA que con mayor frecuencia están entre las que presentan una menor mortalidad global.

Los resultados del análisis han puesto de relieve desigualdades en el estado de salud percibido, el uso y el desempeño de los servicios sanitarios, y la mortalidad, tanto entre CCAA como entre géneros y clases sociales, y contribuyen a generar evidencia sobre la magnitud del problema en nuestro país y los posibles beneficios que pueden derivarse de su estudio. Esta información puede ser la base para el desarrollo de políticas posteriores dirigidas a la reducción de desigualdades, tanto desde el punto de vista de la salud pública y la política sanitaria, como de otras políticas sociales (31).

\section{Agradecimientos}

Este estudio se integra en la línea de estudios comparados de resultados en salud asignada al Observatorio del SNS, en el ámbito de las políticas de equidad y cohesión social, y ha sido parcialmente financiado por el Observatorio.

\section{Bibliografía}

[1] BORRELL C, PASARÍN MI. Desigualdad en salud y territorio urbano. Gac Sanit. 2004;18(1):1-4.

[2] REGIDOR E, GUTIÉRREZ-FISAC JL, RODRÍGUEZ C. Diferencias y desigualdades en salud en España. Madrid: Díaz de Santos; 1994.

[3] NAVARRO V, BENACH J y la Comisión científica de estudios de las desigualdades sociales en salud en España. Desigualdades sociales en salud en España. Madrid: Ministerio de Sanidad y Consumo; 1996.

[4] BORRELL C, FERNÁNDEZ E, SCHIAFFINO A, BENACH J, RAJMIL L, VILLALBÍ JR, SEGURA A. Social class inequialities in the use of and access to health services in Catalonia, Spain: what is the influence of supplemental private health insurance? Int J Qual Health Care. 2001;13(2):117-125.

[5] RAJMIL L, BORRELL C, STARFIELD B, FERNANDEZ E, SERRA V, SCHIAFFINO A, SEGURA A. The quality of care and influence of double health care coverage in Catalonia (Spain). Arch Dis Child. 2000;83(3):211-4. 
[6] REGIDOR E, DE MATEO S, GUTIÉRREZ-FISAC JL, FERNÁNDEZ DE LA HOZ K, RODRÍGUEZ C. Diferencias socioeconómicas en la utilización y accesibilidad de los servicios sanitarios en España. Med Clin (Barc). 1996;107:285-8.

[7] FERNÁNDEZ E, SCHIAFFINO A, RAJMIL L, BADIA X, SEGURA A. Gender inequalities in health and health care services use in Catalonia (Spain). J Epidemiol Community Health. 1999;53:218-22.

[8] ROHLFS I. Diferencias y desigualdades. La salud de hombres y mujeres en la ciudad de Barcelona. Barcelona: Universitat Autònoma de Barcelona; 1998.

[9] World Health Organization. The World Health Report 2000: Health Systems Performance. Geneve: World Health Organization; 2000.

[10] National Health Service. NHS Performance Indicators. National Figures: February 2002. London: National Health Service; 2003.

[11] GARCÍA-ALTÉS A, ZONCO L, BORRELL C, PLASENCIA A. Measuring the performance of health care services: international experiences and their application to an urban context. Gac Sanit. 2006;20(4):316-24.

[12] MANNION R, GODDARD M. Performance measurement and improvement in health care. Appl Health Econ Health Policy. 2002;1(1):13-23.

[13] MURRAY CJL, FRENK J. A framework for assessing the performance of health systems. Bull World Health Organ. 2000;78(6):811-20.

[14] RODRÍGUEZ-SANZ M, BORRELL C, URBANOS R, PASARÍN MI, RICO A, FRAILE M, RAMOS X, NAVARRO V. Power relations and premature mortality in Spain's Autonomous Communities. Int J Health Serv. 2003;33:687-722.

[15] BENACH J, YASUI Y, BORRELL C, PASARIN MI, MARTÍNEZ JM, DAPONTE A. The public health burden of material deprivation: excess mortality in leading causes of death in Spain. Prev Med. 2003;36:300-8.

[16] BORRELL C, PLASENCIA A, PASARIN I, ORTUN V. Widening social inequalities in mortality: the case of Barcelona, a southern European city. J Epidemiol Community Health. 1997;51(6):659-67.

[17] BORRELL C, ARIAS A. Socioeconomic factors and mortality in urban settings: the case of Barcelona, Spain. J Epidemiol Community Health. 1995;49(5):460-65.

[18] BORRELL C, REGIDOR E, ARIAS LC, NAVARRO P, PUIGPINOS R, DOMINGUEZ V, PLASENCIA A. Inequalities in mortality according to educational level in two large Southern European cities. Int J Epidemiol. 1999;28(1):58-63.

[19] REGIDOR E, DE MATEO S, GUTIÉRREZ FISAC JL, RODRÍGUEZ C. Diferencias socioeconómicas en mortalidad en 8 provincias españolas. Med Clin. 1996; 106:285-289.

[20] BENACH J, BORRELL C, GARCÍA MD, CHAMIZO H. Desigualdades sociales en mortalidad en áreas pequeñas en España. En: La salud pública y el futuro del estado del bienestar. Informe SESPAS 1998. Granada: Escuela Andaluza de Salud Pública; 1998.

[21] BENACH J, YASUI Y, BORRELL C, ROSA E, PASARIN MI, BENACH N, ESPAÑOL E, MARTÍNEZ JM, DAPONTE A. Atlas de mortalidad en áreas pequeñas en España (1987-1995). Barcelona: Universitat Pompeu Fabra; 2001.

[22] ABAD DIEZ JM, CARRETER ORDÓÑEZ C. Indicadores sanitarios por Comunidades Autónomas. En: Invertir para la salud. Prioridades en salud pública. Informe SESPAS 2002. Valencia: Escuela Valenciana de estudios para la Salud; 2002. 
[23] BORRELL C, BENACH J (Grupo de trabajo CAPS-F. J. Bofill). La evolución de las desigualdades en salud en Cataluña. Gac Sanit. 2006;20(5):396-406.

[24] BORRELL C, GARCIA-CALVENTE M, MARTÍ-BOSCÁ JV (eds.). Informe de la Sociedad Española de Salut Pública y Adminsitración Sanitaria 2004: La salud pública desde la perspectiva de género y clase social. Gac Sanit; 2004;18 Suppl $1: 2-6$

[25] REGIDOR E, GUTIERREZ-FISAC JL, DOMÍNGUEZ V, CALLE ME, NAVARRO P. Comparing social inequalities in health in Spain: 1987 and 1995/97. Soc Sci Med. 2002;54(9):1323-32.

[26] RODRÍGUEZ-SANZ M, CARRILLO-SANTISTEVE P, BORRELL C. Desigualdades sociales en la salud, los estilos de vida y la utilización de servicios sanitarios en las CCAA 1993-2003. Observatorios de salud de la mujer y del SNS. Agencia de Calidad. Ministerio de Sanidad y Consumo. 2005.

[27] GARCÍA-ALTÉS A, FERRANDO J, MENDIVIL J, BORRELL C. Análisis de las desigualdades de género y clase social en el desempeño de los servicios sanitarios de las Comunidades Autónomas. Observatorios de salud de la mujer y del SNS. Agencia de Calidad. Ministerio de Sanidad y Consumo. 2005.

[28] PÉREZ G, CIRERA E, RODRÍGUEZ M, BORRELL C. Desigualdades de mortalidad en las Comunidades Autónomas entre 1981 y 2002. Observatorios de salud de la mujer y del SNS. Agencia de Calidad. Ministerio de Sanidad y Consumo. 2005.

[29] Grupo de trabajo de la SEE y de la SEMFYC. Una propuesta de medida de la clase social. Aten Primaria. 2000;25:350-63.

[30] WHO Regional Office for Europe. The European health report 2002. Copenhagen: WHO Regional Office for Europe ; 2002. WHO Regional Publications, European Series, No. 97

[31] REGIDOR E, DOMINGUEZ V, NAVARRO P, RODRIGUEZ C. The magnitude of differences in perceived general health associated with educational level in the regions of Spain. J Epidemiol Community Health. 1999;53(5):288-93.

[32] BORRELL C, PASARÍN MI. The study of social inequalities in health in Spain: where are we? J Epidem Community Health. 1999;53:388-9. 
